ARTICLE

https://doi.org/10.1038/s41467-019-14016-1

\title{
Reaction scope and mechanistic insights of nickel-catalyzed migratory Suzuki-Miyaura cross-coupling
}

Yuqiang $\mathrm{Li}^{1}$, Yixin Luo ${ }^{2}$, Long Peng ${ }^{1}$, Yangyang $\mathrm{Li}^{1}$, Binzhi Zhao', Wang Wang${ }^{1}$, Hailiang Pang ${ }^{1}$, Yi Deng ${ }^{1}$, Ruopeng $\mathrm{Bai}^{2}, \mathrm{Yu} \operatorname{Lan}^{2,3 \star} \&$ Guoyin Yin (1D) ${ }^{1 \star}$

Cross-coupling reactions have developed into powerful approaches for carbon-carbon bond formation. In this work, a Ni-catalyzed migratory Suzuki-Miyaura cross-coupling featuring high benzylic or allylic selectivity has been developed. With this method, unactivated alkyl electrophiles and aryl or vinyl boronic acids can be efficiently transferred to diarylalkane or allylbenzene derivatives under mild conditions. Importantly, unactivated alkyl chlorides can also be successfully used as the coupling partners. To demonstrate the applicability of this method, we showcase that this strategy can serve as a platform for the synthesis of terminal, partially deuterium-labeled molecules from readily accessible starting materials. Experimental studies suggest that migratory cross-coupling products are generated from $\mathrm{Ni}(\mathrm{O} / \mathrm{II})$ catalytic cycle. Theoretical calculations indicate that the chain-walking occurs at a neutral nickel complex rather than a cationic one. In addition, the original-site cross-coupling products can be obtained by alternating the ligand, wherein the formation of the products has been rationalized by a radical chain process.

\footnotetext{
${ }^{1}$ Institute for Advanced Studies, Wuhan University, Wuhan 430072 Hubei, PR China. ${ }^{2}$ School of Chemistry and Chemical Engineering, Chongaing Key Laboratory of Theoretical and Computational Chemistry, Chongqing University, Chongqing 400030, PR China. ${ }^{3}$ College of Chemistry and Molecular Engineering, Zhengzhou University, Zhengzhou 450001, PR China. *email: lanyu@cqu.edu.cn; yinguoyin@whu.edu.cn
} 
T ransition metal-catalyzed cross-coupling reactions have developed into powerful approaches for carbon-carbon bond formation, and have revolutionized synthetic strategies in medicinal chemistry and material science ${ }^{1-5}$. Historically, the successful cross-coupling of alkyl electrophiles represents a landmark of this research field ${ }^{6-9}$. The $\beta$-hydrogen-containing, electronically unactivated alkyl partners is generally recognized to be more challenging than their aryl and vinyl analogs ${ }^{10-12}$. Over the past two decades, significant effort has been devoted to avoiding $\beta$-hydride elimination and constructing chemical bonds at the original position in an efficient and selective manner ${ }^{13-16}$. Chain-walking highlights great opportunities in bond formation at new positions, the exploration of transformations involving chain-walking have stimulated considerable interest in the synthetic community recently ${ }^{17,18}$. In this context, the concept of migratory cross-coupling has been initially described by the Baudoin group in palladium-catalyzed $\mathrm{C}-\mathrm{H}$ bond functionalization reactions (Fig. 1a) ${ }^{19-21}$.

Recently, it has been reported that nickel catalysis ${ }^{22}$ exhibits unique performance in migratory transformations ${ }^{23}$. Particularly, a set of reductive migratory cross-coupling with aryl halides have been disclosed by $\mathrm{Zhu}^{24,25}$ and our group ${ }^{26,27}$ independently, wherein a benzylic selectivity is achieved and a series of pharmaceutically relevant 1,1-diarylalkanes can be efficiently constructed (Fig. 1b). In the past decade, extensive efforts have been devoted to the mechanistic studies of nickel-catalyzed crosscoupling of alkyl electrophiles ${ }^{33,14,28-30}$. While the well-known $\mathrm{Ni}$ $(0 / \mathrm{II})^{10,31,32}$ or $\mathrm{Ni}(\mathrm{II} / \mathrm{IV})^{8,33}$ catalytic cycles are proposed in some cases, a radical chain process and bonding formation from a $\mathrm{Ni}$ (III) intermediate is particularly prevailed in those reactions with a nitrogen-based ligand $29,30,34$. In contrast, there are still quite limited reports on the mechanism of the nickel-catalyzed migratory cross-coupling reactions $\mathrm{s}^{25,35}$. Compared with classical cross-coupling reactions, the major difference of migratory crosscoupling is a metal chain-walking process involved in the catalytic cycle. More elementary steps in catalytic cycle mean more complicated in mechanism, which increases difficulty into the mechanistic investigations as well. As a result, mechanistic information on the details for catalytic cycle, nickel chain-walking process and the origin of regioselectivity and so on, is still scarce, which seriously restricts further reaction design.

Furthermore, the current methods have several limitations in their substrate scope. First, in our previous investigations, the aryl coupling partners were restricted to electron-neutral and -rich aryl bromides ${ }^{26,27}$. Second, functionalization at benzylic position after chain-walking has been well-studied, but the synthetically useful allylic positions are unachievable ${ }^{24,26}$. Besides, the use of inexpensive, abundant nucleophiles for a redox-neutral migratory coupling reaction remains underdeveloped. For example, the migratory Suzuki-Miyaura cross-coupling of alkyl electrophiles has only been demonstrated by Sigman with palladium catalysis, wherein only allylic products and 1,2-palladium migration could

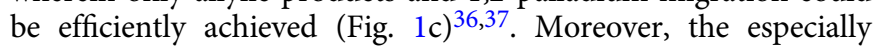
useful, unactivated alkyl chlorides ${ }^{38-40}$ have not been successfully employed in a migratory coupling event to date.

Here, we report our work on developing the general, $\mathrm{Ni}$ catalyzed migratory Suzuki-Miyaura cross-coupling of unactivated alkyl electrophiles with both aryl and vinyl boron reagents (Fig. 1d). Experimental and computational studies are performed to reveal the detailed mechanism.

a Migratory cross-coupling of alkyl electrophiles

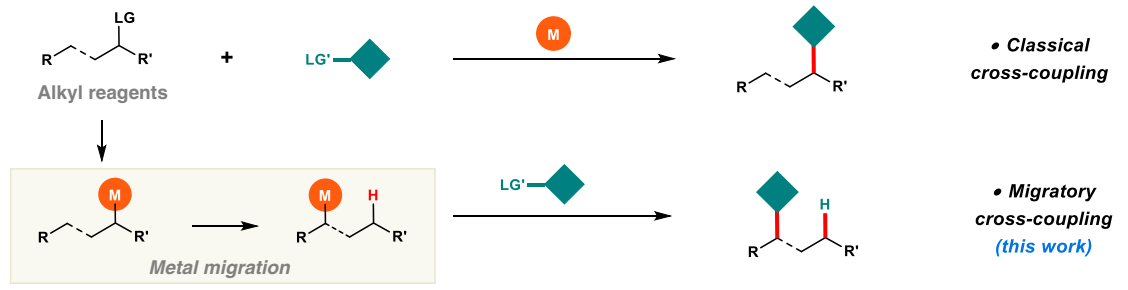

b Ni-catalyzed reductive migratory cross-coupling (Zhu and our group):

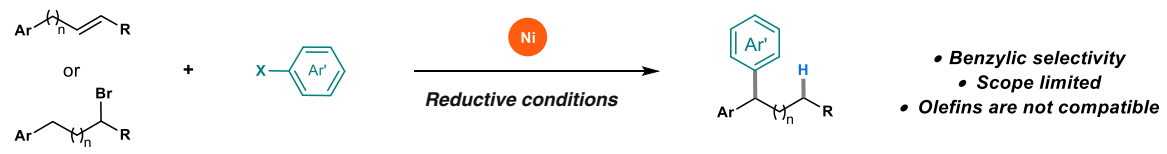

C Pd-catalyzed migratory suzuki-miyaura cross-coupling (Sigman):

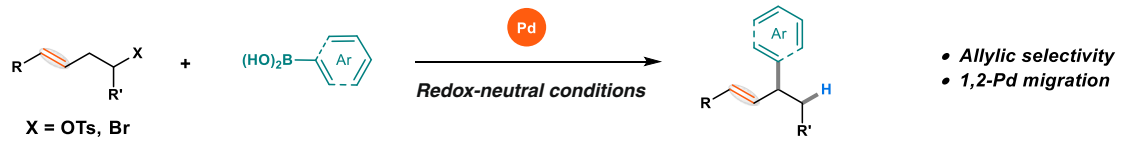

d Ni-catalyzed migratory suzuki-miyaura cross-coupling (this work):
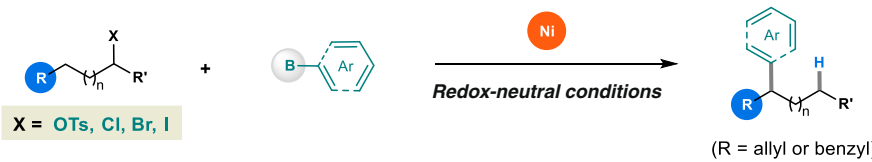
- Allylic \& benzylic selecitivity
- Olefins are compatible
- Up to 1,7-Ni migration
- Mechanism study

Fig. 1 Transition metal-catalyzed migratory cross-coupling. a Migratory cross-coupling of alkyl electrophiles. b Ni-catalyzed reductive migratory crosscoupling. c Pd-catalyzed migratory Suzuki-Miyaura cross-coupling. $\mathbf{d}$ The approach developed in this study. 
Table 1 Reaction optimizationa.

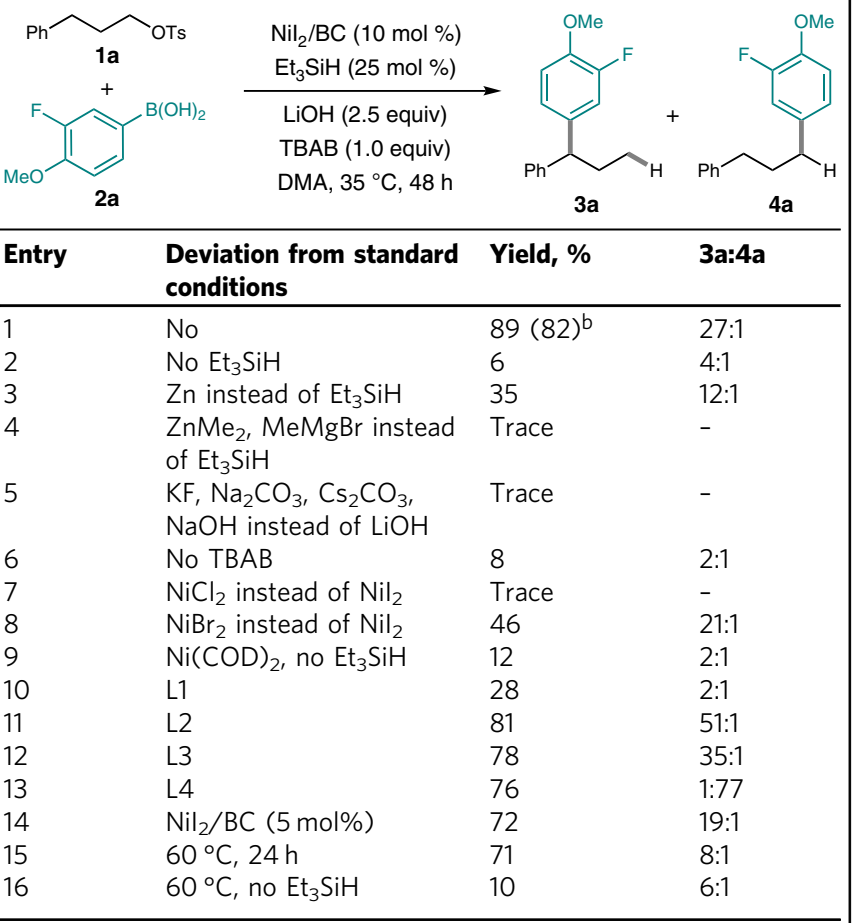

aStandard conditions: $\mathrm{Nil}_{2}(15.6 \mathrm{mg}, 0.05 \mathrm{mmol}, 10 \mathrm{~mol} \%), \mathbf{B C}(18.0 \mathrm{mg}, 0.05 \mathrm{mmol}, 10 \mathrm{~mol} \%$ ), $\mathrm{Et}_{3} \mathrm{SiH}(20 \mu \mathrm{L}, 0.13 \mathrm{mmol}, 25 \mathrm{~mol} \%), \mathbf{1 a}(145.0 \mathrm{mg}, 0.5 \mathrm{mmol}, 1.0$ equiv), $\mathbf{2 a}(127.5 \mathrm{mg}$, $0.75 \mathrm{mmol}, 1.5$ equiv), LiOH (29.9 mg, $1.25 \mathrm{mmol}, 2.5$ equiv), TBAB $(161.2 \mathrm{mg}, 0.5 \mathrm{mmol}, 1.0$ equiv), DMA $(4 \mathrm{~mL})$. Yields were determined by $\mathrm{GC}$ with 1,3,5-trimethoxybenzene as the internal standard

b/solated yield

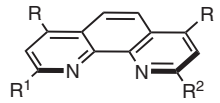

BC: $R=P h, R^{1}=R^{2}=M e$ L1: $R=P h, R^{1}=M e, R^{2}=H$ L2: $R=H, R^{1}=R^{2}=M e$

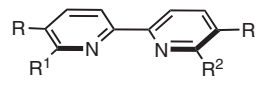

L3: $R=H, R^{1}=R^{2}=M e$ L4: $R=M e, R^{1}=R^{2}=H$

\section{Results}

Reaction optimization. We launched the investigation with the cross-coupling of 3-phenylpropyl tosylate (1a) with aryl boronic acid $\mathbf{2 a}$. The preliminary study demonstrated that the generation of active nickel catalyst was not efficient via reduction of $\mathrm{Ni}(\mathrm{II})$ salts by $\mathbf{2 a}$. A series of $\mathrm{Ni}(\mathrm{II})$ precatalyst activation methods were tested in the following studies ${ }^{41}$. The addition of $25 \mathrm{~mol} \% \mathrm{Et}_{3} \mathrm{SiH}$, proved to be an efficient method to generate the active nickel catalyst, which offering the coupled products in $82 \%$ isolated yield (Table 1, entries 1-5, and Supplementary Fig. 3). Base is also crucial to the success of this transformation. The importance of $\mathrm{LiOH}$ was highlighted by the failure of other bases which only afford trace amount product (Table 1, entry 5, and Supplementary Table 1).

The addition of TBAB greatly improves the efficiency, which due to the in situ conversion of the alkyl tosylate into alkyl bromide (Table 1, entry 6). Nickel iodide is superior to other nickel precatalysts (Table 1, entries 7-9). Dimethyl substitution on 1,10-phenanthroline or 2,2'-bipyridine backbones are crucial for the migratory selectivity (Table 1 , entries 10-13). Notably, the original-site Suzuki-Miyaura cross-coupling ${ }^{42,43}$ product 4 a could also be obtained in a good yield and excellent selectivity when the $5,5^{\prime}$-dimethylbipyridine (L4) was used (Table 1, entry 13 ), which is consistent with our previous reductive cross-couplings ${ }^{26}$. Solvent evaluation indicated that only amide solvents could deliver the migratory product 3a (Supplementary Table 1). Decreasing the catalyst loading to $5 \mathrm{~mol} \%$ resulted in moderately lower yield and selectivity (Table 1, entry 14). Increasing the temperature to $60^{\circ} \mathrm{C}$ led to worse selectivity (Table 1, entries 15 and 16).

Substrate scope. With the optimal conditions in hand, we next shifted our attention to investigating the generality of the Nicatalyzed migratory Suzuki-Miyaura cross-coupling reaction. First, the scope of alkyl tosylates, which are readily accessible from the corresponding alcohols, was examined. As shown in Fig. 2 (top part), all examples furnished the migratory coupling products in good to excellent yields and regioselectivity under the standard conditions. A class of 1,1-diarylalkanes was prepared accordingly, which are widely encountered in biologically relevant molecules ${ }^{44,45}$. To our delight, both electronic-rich and deficient aryl coupling partners performed smoothly under this redoxneutral conditions. Moreover, homoallyl (3v) and homostyrenyl tosylates were also suitable substrates for this nickel-catalyzed system. Vinyl (3m and $3 \times$ ) and styrenyl (3w, 3ae-3ag) boronic acids coupled with the homostyrenyl tosylates, to provide the skipped diene products with high regioselectivity $(\mathbf{3} \times, \mathbf{3 a e}-\mathbf{3 a g})$. It should be noted that in Knochel's study, an olefin coordination with nickel could facilitate reductive elimination at the original position ${ }^{32}$.

The scope of the nickel-catalyzed migratory reaction could also be extended to alkyl halides. As illustrated in Fig. 2 (3ah-3e), both primary and secondary alkyl bromides were able to yield the benzylic coupling products efficiently under the conditions without TBAB. The tertiary bromide was also able to provide the migratory product, albeit in low yield under the current conditions (3ap). The coupling reaction could also proceed when the iodide analog was employed, but with poor regioselectivity under the same conditions (3as). Gratifyingly, the more challenging chloride electrophiles were also converted to the benzylic arylation products efficiently in the presence of KI under an elevated temperature $\left(80^{\circ} \mathrm{C}\right)$ (Supplementary Table 2), which dramatically broadens the application of this migratory strategy in cascade or iterative synthesis with polyhalogenated materials ${ }^{46}$. In addition, a gram-scale experiment showed the scalability of this method (3aj). All 1,2- to $1,7-\mathrm{Ni}$ migration (3s) coupling products could be successfully accessed under the redox-neutral conditions. Finally, a range of functional groups such as trimethylsilyl $\left(\mathrm{SiMe}_{3}\right)$, ester $\left(\mathrm{CO}_{2} \mathrm{Me}\right)$, amines $\left(\mathrm{NMe}_{2}, \mathrm{NPh}_{2}\right)$, thioether (SMe), cyano $(\mathrm{CN})$, double bonds $(\mathrm{C}=\mathrm{C})$, pyridinyl, and quinolyl were well tolerated in this transformation. Notably, the double bonds can survive in this protocol makes it different from the strategy of using olefin and stoichiometric silanes as the susbstrates ${ }^{25}$.

Aryl boronic esters could also be employed as the coupling partner to deliver the migratory coupling products (Fig. 3a). It is noteworthy that 3,5-disubstituted aryl boronic esters can be prepared directly from the corresponding arenes by a highly efficient iridium-catalyzed borylation ${ }^{47}$.

In addition, the scope of the original-site Suzuki-Miyaura cross-coupling of alkyl bromides with aryl boronic acids was also studied. As shown in Fig. 3b, the electronic property of the aryl boronic acids did not affect the reactivity. Both primary and secondary alkyl bromides could successfully yield the corresponding cross-coupling products. However, tertiary alkyl bromide shows no reactivity. It should be noted that the migratory benzylic cross-coupling products were also detected in most cases, but no terminal coupling products were observed in the reactions with secondary alkyl bromides due to the limitation of our instruments. 

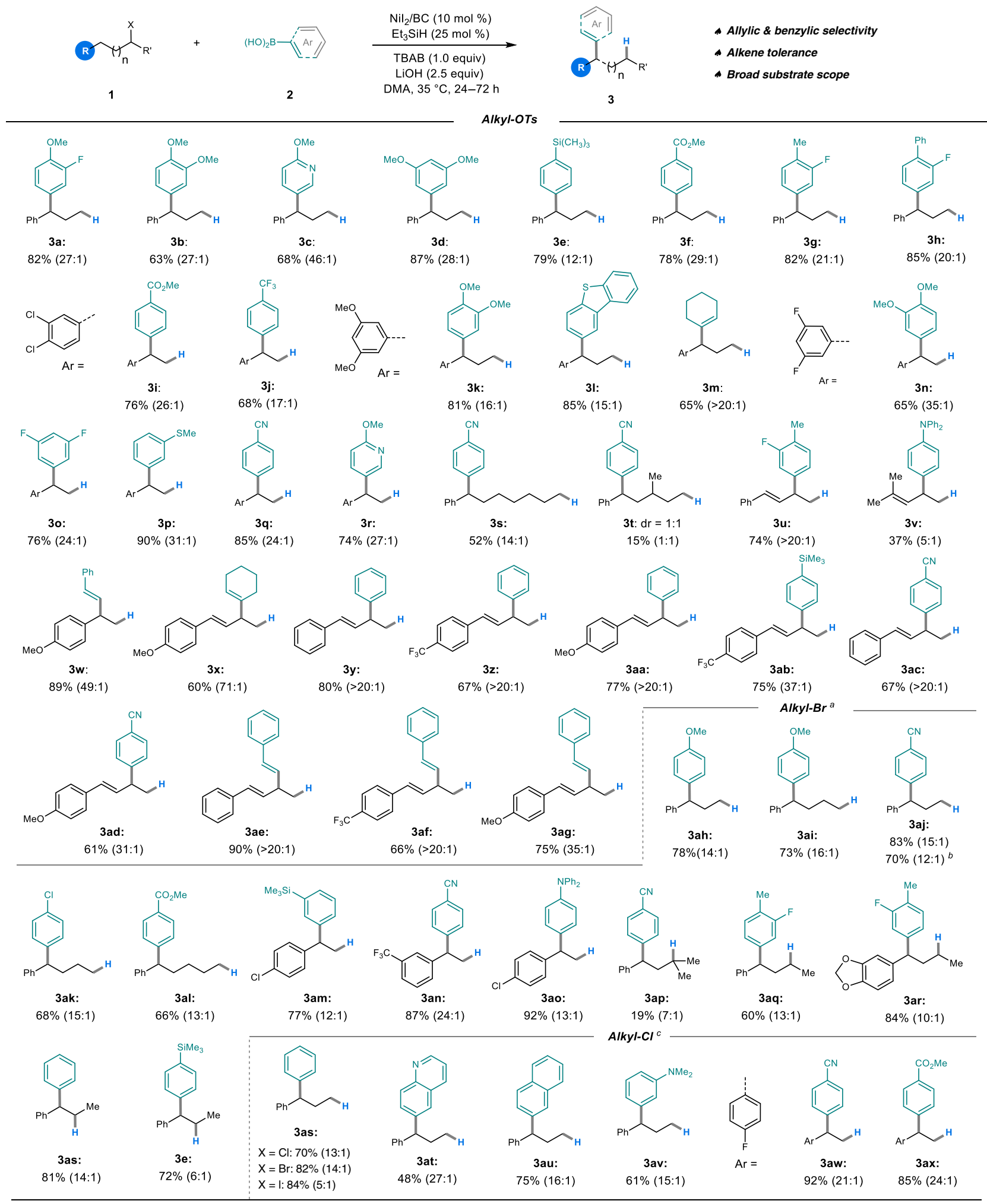

Fig. 2 Scope of the methodology. Isolated yields were shown. Ratios of $\mathbf{3 : 4}$ are determined by GCMS. aWithout TBAB Additive. b10 mmol scale. ${ }^{\mathrm{C} K \mathrm{I}}$ ( $0.38 \mathrm{mmol}, 0.75$ equiv), $\mathrm{LiOH}\left(0.75 \mathrm{mmol}, 1.5\right.$ equiv), the mixture was stirred at $80^{\circ} \mathrm{C}$.

Mechanism discussion. The application of nickel catalyst constitutes a great advance in the cross-coupling of alkyl electrophiles $^{8,13}$. The nitrogen-based ligands enable nickel-catalyzed reactions prefer to go through single-electron transfer (SET) processes, which increases the difficulty in mechanism investigation. Although preliminary mechanistic studies towards nickel- catalyzed migratory cross-coupling have been done by Zhu's ${ }^{24}$ and our group ${ }^{26}$, several important concerns are still unclear, e.g., the origin of regioselectivity, the electronic character of nickel catalyst in chain-walking process and why olefins can dissociate from the catalyst during chain-walking, etc. This homogenous, redox-neural cross-coupling reaction provides a good platform to 
a

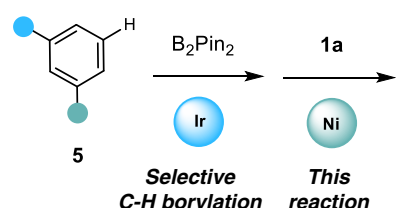

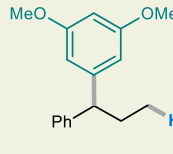

$3 d$

$58 \%(13: 1)$

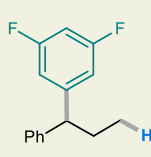

3ay:

$61 \%(11: 1)$ b
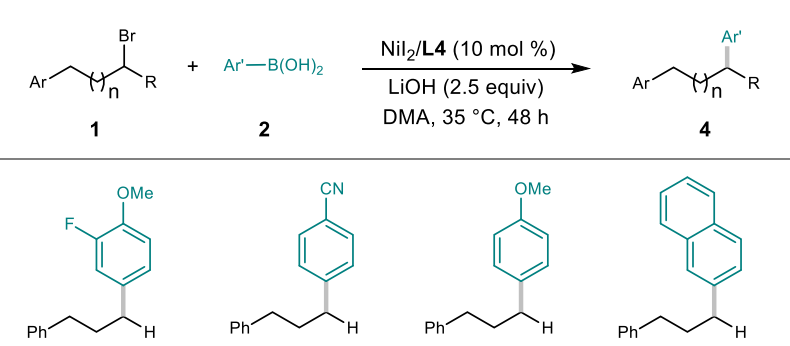

4a: $83 \%(45: 1)$

4aj: $84 \%(34: 1)$

4ah:
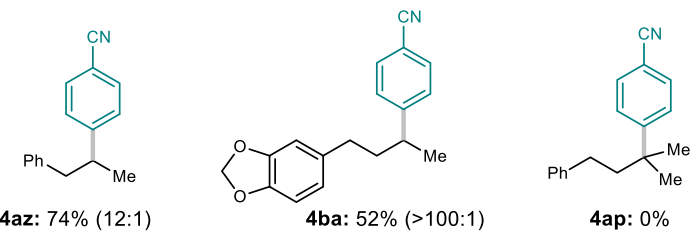

4az: $74 \%(12: 1)$

4ap: $0 \%$

Fig. 3 Expansion of substrate scope. a Migratory Suzuki-Miyaura crosscoupling of aryl borate. b Original-site Suzuki-Miyaura cross-coupling.

obtain insights into the details of chain-walking, which would supply a rational basis for the further development of transformations involving nickel migration.

Moreover, this Suzuki-Miyaura reaction and our prior reductive condition reactions share many similarities, such as the same ligands, solvent, temperature, and the same selectivity (Fig. 4a). These similarities imply that these two type reactions share some commences in mechanism. The mechanistic study from the redox-neutral reaction can help us to understand the more complicated reductive ones.

Even though significant achievements have been made in mechanistic studies on nickel-catalyzed cross-coupling of alkyl electrophiles with an array of metal reagents ${ }^{48,49}$ or another electrophiles ${ }^{14}$. However, reports on systematical mechanistic studies on the nickel-catalyzed Suzuki-Miyaura cross-coupling of alkyl electrophiles are still limited to date ${ }^{50,51}$.

As a result, after achieving this nickel-catalyzed migratory Suzuki-Miyaura cross-coupling reaction, we started to investigate its mechanism.

First of all, to gain additional insight into the nickel migration, deuterium-labeled tosylates were synthesized and examined in this reaction. First, the reaction of $\mathbf{1 a}-\mathrm{D}_{2}$ with $\mathbf{2} \mathbf{b}$ furnished the D-migrated product $3 \mathbf{b b}-\mathrm{D}_{2}$ with $100 \%$ benzylic deuterium retention and $63 \% \mathrm{D}$-incorporation at the homobenzylic position (Fig. 4b-1). These results suggest that the formation of migratory coupling products involve a key step of nickel chain-walking. Besides, the reaction of $1 \mathbf{a}-\mathrm{D}_{2}$ ' with $\mathbf{2} \mathbf{c}$ delivered the migratory product 3as- $\mathrm{D}_{2}$ ' with $98 \%$ deuterium retention under the standard conditions (Fig. 4b-2). These observations reflect that this method can serve as a platform to synthesize terminal partially D-labeled compounds. Deuterium-labeled molecules have been drawing increasing attention in medicinal chemistry recently ${ }^{52,53}$, and partially D-labeled methyl groups $\left(\mathrm{CDH}_{2}\right.$ and $\mathrm{CD}_{2} \mathrm{H}$ ) were demonstrated to be useful in spectroscopic investigations and metabolic studies ${ }^{54}$. As a consequence, a series of $2 \mathrm{D}$ and 1D-labeled 1,1-diarylalkane derivatives were

prepared from the readily accessible $\alpha-D$ substituted tosylates (Fig. 4c).

Next, only trace amount of cross-coupling products were detected under the standard conditions when an alkyl bromide without an aryl group substituent (6a) was employed (Fig. 4d). This finding inspires us to investigate the role of the aryl group on the alkyl electrophiles. Further study found that using an electron-deficient olefin L5 as ligand, the yield of the crosscoupling products could be improved to 30\% (Fig. 4d). These results are agreed with the fact that reductive elimination from $\mathrm{Ni}$ (II) species is difficult, which can be promoted by the electrondeficient olefin ligands 32,55 . Therefore, the aryl group plays a significant role of promoting the reductive elimination.

Consequently, a catalytic cycle involving an alkyl-Ni(II)-Ar intermediate is proposed to rationalize this Suzuki-Miyaura reaction. As depicted in Fig. 4e, inefficient reductive elimination of the $\mathrm{Ni}(\mathrm{II})$ complex III leads to the formation of $\beta-\mathrm{H}$ elimination complex IV. Subsequent reductive elimination generates an arene (8) and an olefin (9).

The corresponding deborylation and olefin side products in the reaction of 6 a with $2 c$ were detected under our optimal conditions. A deuterium-labeled isopropyl bromide $\left(\mathbf{6} \mathbf{b}-\mathbf{D}_{\mathbf{6}}\right)$ was examined under the standard conditions, the deborylation product (8b) was isolated in $47 \%$ yield with $31 \%$ D-

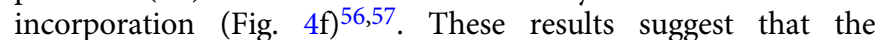
hydrogen atom of the deborylation product is partly from the alkyl electrophile, which agrees with the proposed catalytic cycle (Fig. 4e) and reveals that the aryl group of the alkyl electrophile plays the role of promoting the carbon-carbon bond formation at benzylic position.

In addition, several reports demonstrated the possibility of reductive elimination from the $\mathrm{Bn}-\mathrm{Ni}$ (II)-Ar intermediates to construct diarylalkanes ${ }^{58}$, and a very recent report from the $\mathrm{Fu}$ group experimentally established the feasibility of $\mathrm{Ni}(\mathrm{II})$ chainwalking ${ }^{59}$. Based on these results, we proposed a catalytic cycle for the Ni-catalyzed migratory Suzuki-Miyaura reaction. As illustrated in Fig. $4 \mathrm{~g}$, the reaction is initiated by a $\mathrm{Ni}(0)$ species (I), which generates an alkyl-nickel(II) intermediate (III) by stepwise oxidative addition. There are two possibilities in the following steps: (A) a rapid chain-walking occurs to form a thermodynamically stable benzylic nickel(II) complex (V), and following transmetalation with a boronic acid to generate a benzyl-Ni(II)-Ar intermediate (VI); (B) transmetalation occurs before chain-walking, leading to the formation of the intermediate VI as well. Finally, the desired products 3 are produced from VI via reductive elimination.

These two pathways differ in the order of transmetalation and chain-walking. In order to differentiate these two pathways and to obtain details on the catalytic cycle, kinetic studies were conducted next.

Kinetic studies. To simplify the studies, we chose the reaction of alkyl bromide $1 \mathrm{~b}$ with 4 -cyanophenylboronic acid (2e) as a model reaction (Fig. 5a), which does not require the addition of silane. The dependence of the initial rate on nickel catalyst, alkyl bromide, boronic acid, and base concentration was evaluated in each case by monitoring the formation of product by gas chromatography. As illustrated in Fig. 5, a complex kinetic picture with first order in $\mathrm{Ni}$ at low catalyst loading and saturation kinetics at higher loadings was observed (Fig. 5b), possibly indicative of offcycle pathways, which is also observed in a previous report. 60 The reaction exhibits a zeroth-order dependence on the concentration of alkyl bromide (Fig. 5c), and first-order dependence on the concentration of both aryl boronic acid (Fig. 5d) and base (Fig. 5e). 
a

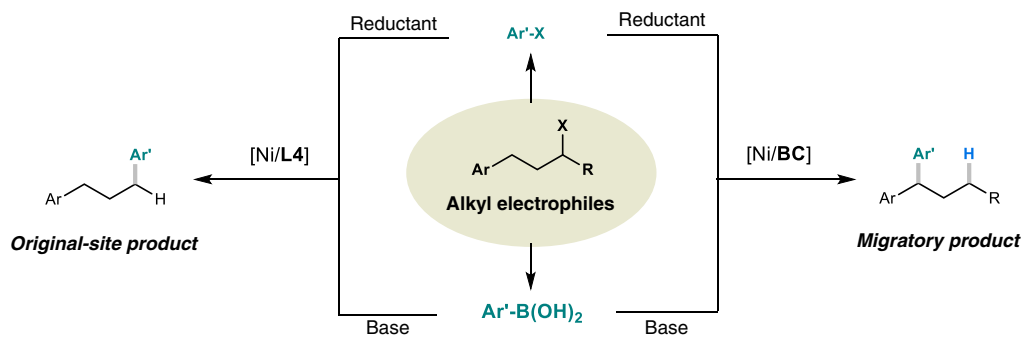

b

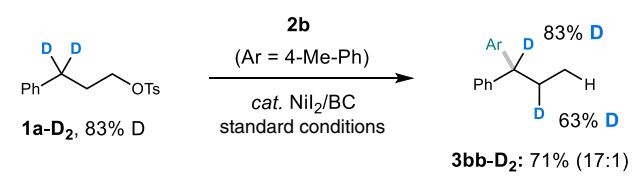

$\overbrace{\text { Ph }}^{\mathrm{D}} \overbrace{\mathrm{OTS}}^{\mathrm{D}}$

$1 \mathrm{a}-\mathrm{D}_{2}{ }^{\prime}, 94 \% \mathrm{D}$
(2)

3as- $D_{2}^{\prime}: 80 \%(27: 1)$

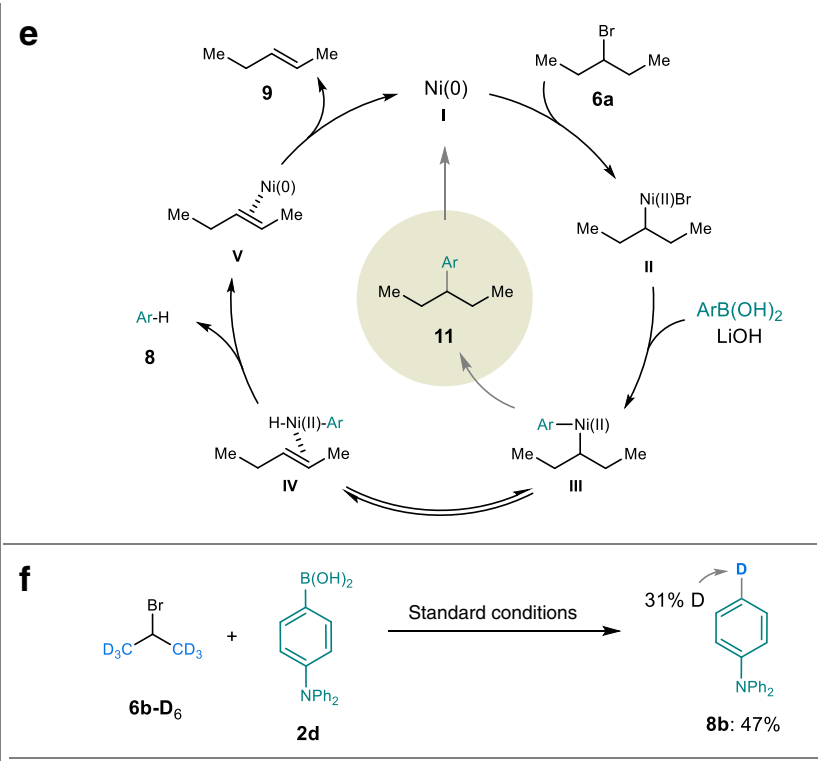

g

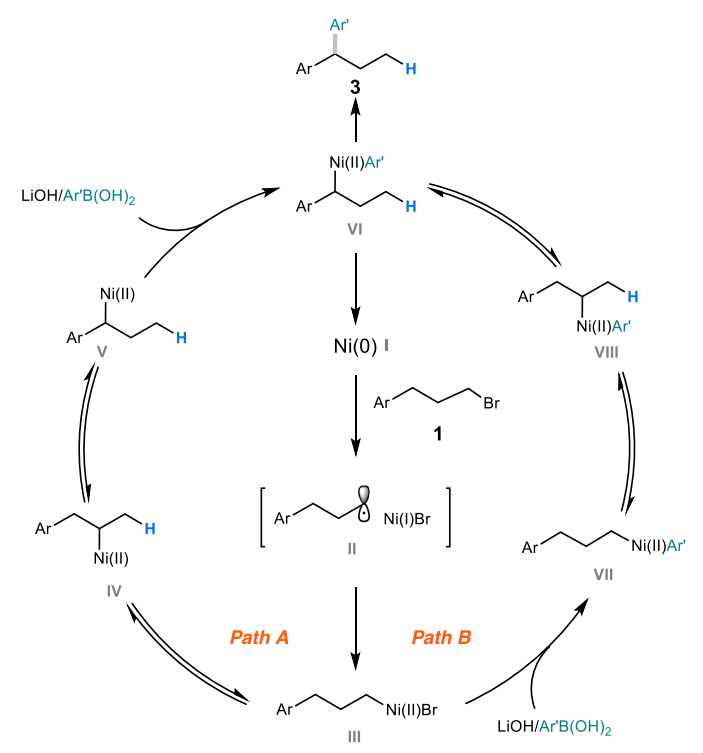

(1)

(2)

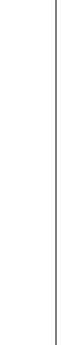

.

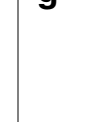

C
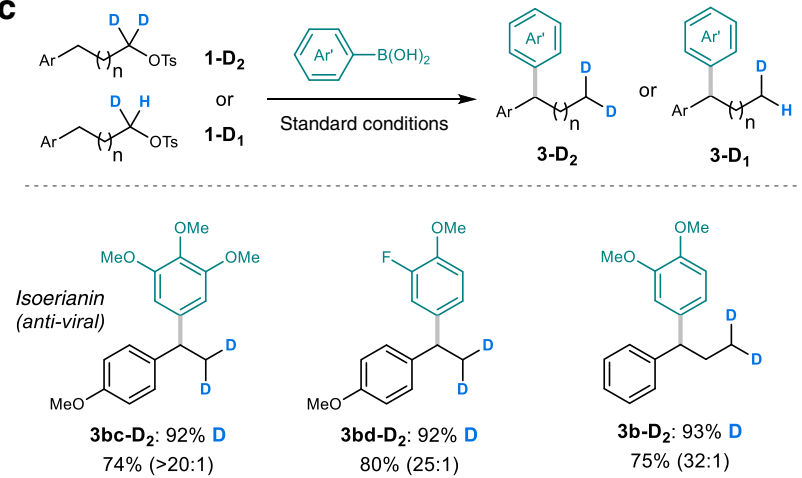

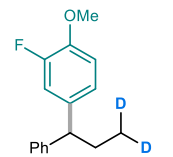

3a- $D_{2}: 90 \%$ D

$72 \%(18: 1)$

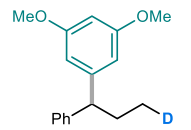

3d-D $1: 86 \%$ D

$76 \%$ (>20:1)

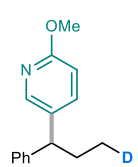

3c-D $: 85 \%$ D $65 \%(>20: 1)$ d

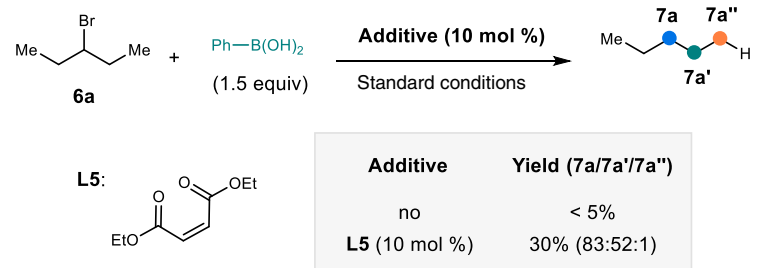

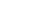

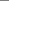




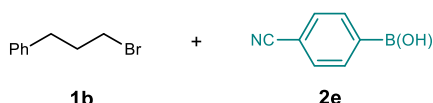

b

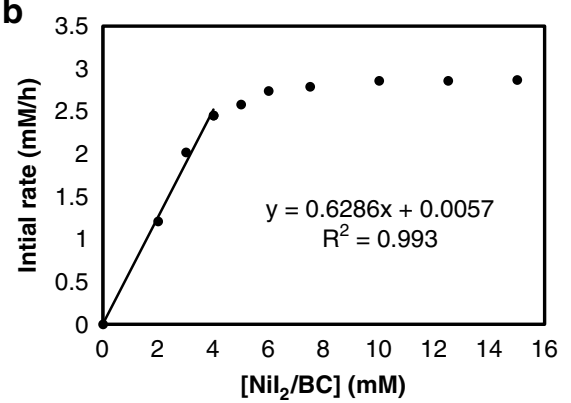

d

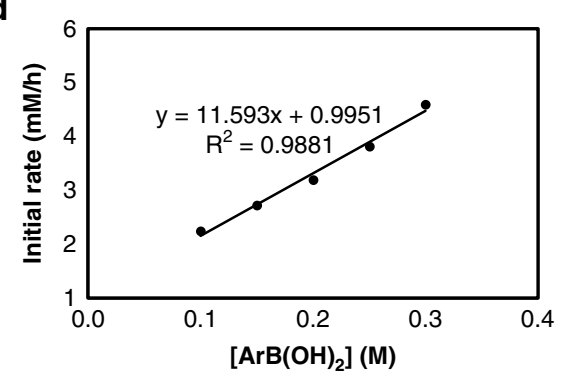

f

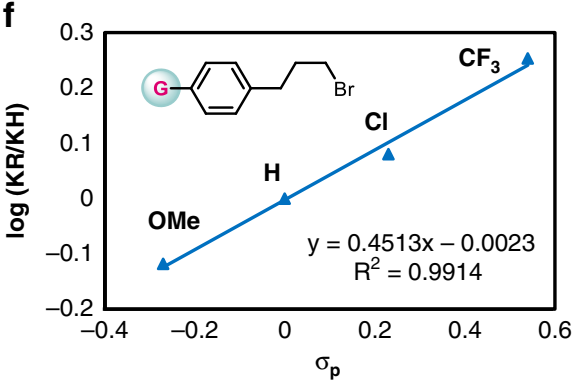

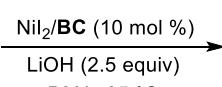

DMA, $35^{\circ} \mathrm{C}$
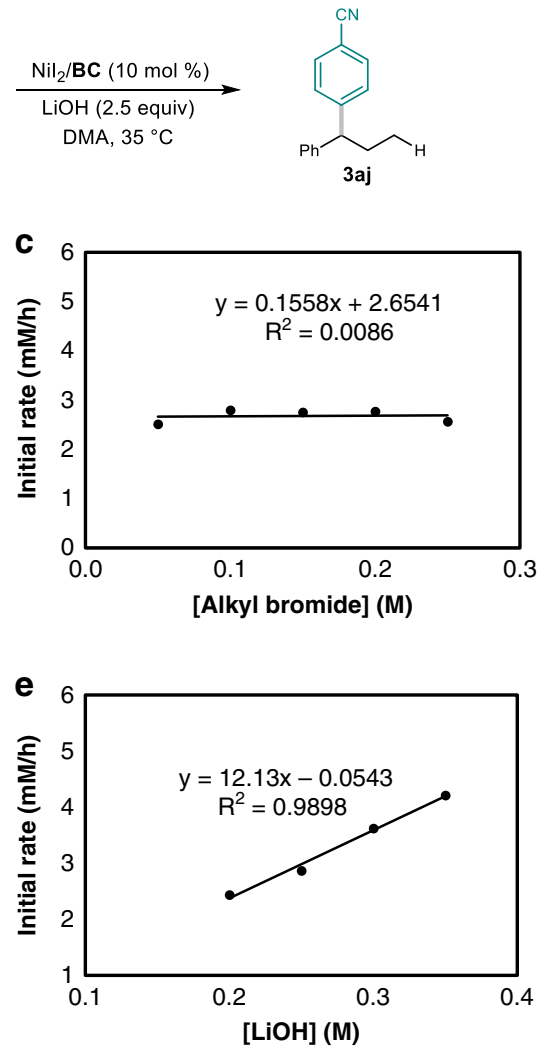

g

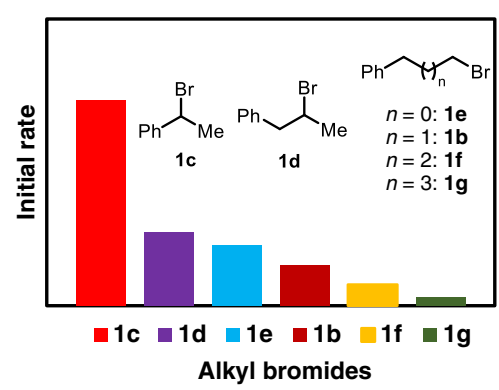

Fig. 5 Kinetic profiles. a Model reaction. b Reaction order in catalyst concentration. c Reaction order in alkyl bromide concentration. $\mathbf{d}$ Reaction order in aryl boronic acid concentration. e Reaction order in base concentration. $\mathbf{f}$ Hammett plots of alkyl bromides. $\mathbf{g}$ Initiate reaction rate of different alkyl bromides.

that the nickel chain-walking occurs prior to transmetalation, which is consistent with the path $\mathrm{A}$, and rules out the path $\mathrm{B}$ (Fig. 4g).

Finally, the initiate rates of different alkyl bromides were also investigated (Fig. 5g). Among of these, secondary benzyl bromide (1c) exhibits the fastest rate, secondary unactivated alkyl bromide (1d) shows faster rate than the corresponding primary one (1e), which indicates the $\mathrm{C}-\mathrm{Br}$ bond cleavage involving a SET process. Moreover, the alkyl bromide with longer carbon chain exhibits slower rate (compare $\mathbf{1 e}$ with $\mathbf{1 b}$, 1f and $\mathbf{1 g}$ ). These results indicate that chain-walking also affects the RDS, which is also agreed with path $\mathrm{A}$ and inconsistent with path $\mathrm{B}$.

Computational studies. Extensive computational studies have been done on chain-walking process in the palladium-catalyzed reactions ${ }^{62}$, in contrast, very limited reports on the nickelcatalyzed ones ${ }^{63}$. In order to get more details on the nickel chainwalking process of this reaction, density functional theory (DFT) calculations were carried out.
With the presence of $\mathbf{B C}$ ligand and reductant additive of $\mathrm{Et}_{3} \mathrm{SiH}$ species, the catalyst precursor $\mathrm{NiI}_{2}$ would generate $\mathrm{Ni}^{0}$ species by ligand exchange and reduction. We first calculated the oxidative addition of alkyl halides with $\mathrm{Ni}(0)$ species $^{64,65}$. As shown in Fig. 6, active catalyst $\mathrm{Ni}(0)$ species $\mathbf{C P 1}$ was chosen as the relative zero point for the free energy profiles, which is coordinated by a BC ligand and one solvent molecule $(N, N$ dimethylacetamide). Subsequent ligand exchange between the alkyl bromide and solvent molecule results in the formation of an intermediate $\mathbf{C P 2}$, with a large energy releasing $(13.6 \mathrm{kcal} / \mathrm{mol})$. The homolytic C-Br bond dissociation via TS1 generates a 3phenylpropyl radical CP4 and an open-shell bromonickel(I) intermediate CP3. The radical rebounds to the nickel intermediate to produce a 3-phenylpropylnickel(II) intermediate CP5. The oxidative addition via a three-membered ring transition state TS2, needs to overcome a barrier of $22.0 \mathrm{kcal} / \mathrm{mol}, 7.5 \mathrm{kcal} / \mathrm{mol}$ higher than that of open-shell $\mathrm{Ni}(0) / \mathrm{Ni}(\mathrm{I})$ pathway. The above results indicate that the alkyl-Ni(II) complex formation via a SET pathway is favorable. 


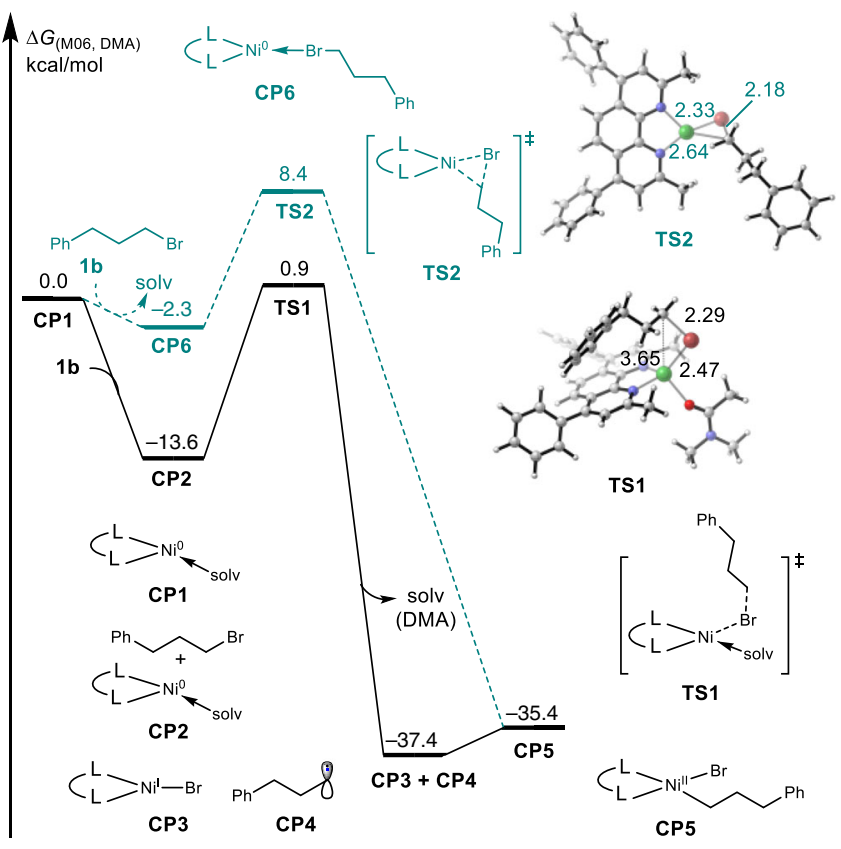

Fig. 6 The calculated reaction energy profile of alkyl-Ni(II) complex formation steps. Energy values are given in $\mathrm{kcal} / \mathrm{mol}$ and represent the relative free energies calculated by the $\mathrm{M06}$ method in $\mathrm{N}, \mathrm{N}$-dimethylacetamide. The values of bond length are given in ångstrom.

After the formation of 3-phenylpropylnickel(II) complex, the following chain-walking process by iterative $\beta$-hydride elimination and migratory insertion were taken into account. As illustrated in Fig. 7 , the first $\beta$-hydride elimination process via TS3 generates a planar $\mathrm{Ni}(\mathrm{II})-\mathrm{H}$ species $\mathrm{CP7}$ and allylbenzene (CP8), which can insert to $\mathrm{Ni}-\mathrm{H}$ bond to form a secondary $\mathrm{C}-\mathrm{Ni}$ (II) species via TS4. Notably, the relative free energy of allylbenzene coordinated $\mathrm{Ni}$ (II) species CP13 is $6.3 \mathrm{kcal} / \mathrm{mol}$ higher than CP7, which suggests that the olefin could dissociate from the nickel complex during the chain-walking process. Subsequent iterative $\beta$-hydride elimination and migratory insertion through TS5 and TS6 finally generates a 1-phenylpropylnickel(II) intermediate CP12. The calculated energy profile shows that the conversion of CP5 to CP12 is a reversible process: the highest energy barrier is first $\beta$-hydride elimination $(14.5 \mathrm{kcal} / \mathrm{mol}$ ) in the forward step (from CP5 to CP12), and last alkene insertion $(16.0 \mathrm{kcal} / \mathrm{mol})$ in the reverse step (from CP12 to CP5), with the same transition state TS3. Both the above two barriers are lower than that of transmetalation step (Fig. 8a, from CP21 to CP25 via the transition state TS13, with a barrier of $22.0 \mathrm{kcal} / \mathrm{mol})$.

In addition, the cationic tetracoordinated nickel chain-walking process was also considered. In the cationic process, the initial state and final state (from CP5 to CP12) are same as the ones in charge neutral process. The highest energy barrier changed to be the last alkene insertion $(24.5 \mathrm{kcal} / \mathrm{mol})$ in the forward process (from CP5 to CP12) and the first $\beta$-hydride elimination (26.0 $\mathrm{kcal} / \mathrm{mol}$ ) in the reverse process (from CP12 to CP5), with the same transition state TS10, which are higher than that in the neutral chain-walking process. Therefore, the cationic nickel chain-walking process is determined to be unfavorable. Energy profile of subsequent transmetalation and $\mathrm{C}-\mathrm{C}$ bond formation is shown in Fig. 8b. Considering charge neutralization and coordination number, we choose the lithium phenylboronate CP20, which is generated via the coordination of hydroxyl to $\mathrm{PhB}$ $(\mathrm{OH})_{2}$, as active boron reagents (See Supplementary Fig. 55 for details) ${ }^{65,66}$. Coordination between 1-phenylpropylnickel(II) intermediate CP12 and lithium phenylboronate compounds CP20 leads to the formation of CP27, which is able to generate a neutral $\mathrm{Ni}(\mathrm{II})$ complex $\mathbf{C P 2 9}$ via a stepwise process involving transition states TS16 (O-Li cleavage and coordinate $\mathrm{Br}-\mathrm{Li}$ bond formation) and TS17 (O-Ni formation and $\mathrm{Ni}-\mathrm{Br}$ cleavage). Subsequent intramolecular transmetalation through a fourmembered ring transition state TS18 generates a phenyl-Ni(II)benzyl intermediate $\mathbf{C P 3 0}$, which is exothermic by $27.0 \mathrm{kcal} / \mathrm{mol}$. The energy barrier of transmetalation after chain-walking is 19.3 $\mathrm{kcal} / \mathrm{mol}$, which is $3.0 \mathrm{kcal} / \mathrm{mol}$ lower than that of direct transmetalation via transition state TS13. Finally, reductive elimination releases migratory product 3as from $\mathbf{C P 3 0}$, via transition state TS19 and with an energy barrier of $14.2 \mathrm{kcal} / \mathrm{mol}$. The RDS is intramolecular transmetalation and the overall activation energy is $19.3 \mathrm{kcal} / \mathrm{mol}$.

First of all, we found that the formation of the original-site coupling product was not concurrent with the migratory product (Fig. 9a, b), in other words, the regioselectivity varied during the reaction conduction (Fig. 9c). This finding reveals that the formation of the two products is probably from different catalytic cycles. Next, a radical probe experiment was carried out. The reaction of 6-bromo-1-hexene (10) with 2e was studied in our reaction conditions. We found that the ratio of uncyclized crosscoupling product $(\mathbf{1 1} \mathrm{U})$ with the 5-exo-cyclized coupling product (11R) gradually increased along with the catalyst loading (Fig. 9d, e). These results indicate that a radical chain process is operating in this transformation, which is in accordance with the reductive cross-electrophiles coupling reactions ${ }^{34}$.

Collectively, a catalytic cycle involving a radical chain process is proposed for the original-site Suzuki-Miyaura cross-coupling reaction. As depicted in Fig. 9f, the reaction is initiated by a $\mathrm{Ni}(\mathrm{II})$ species (I), and an aryl-Ni(II) species (II) is generated after transmetalation. The complex II reacts with an alkyl radical to generate a $\mathrm{Ni}(\mathrm{III})$ intermediate (III), which delivers the product (4) and a $\mathrm{Ni}(\mathrm{I})$ species (IV) via reductive elimination. The $\mathrm{Ni}(\mathrm{I})$ species IV reacts with an alkyl electrophile (1) to yield the alkyl radical and regenerate the $\mathrm{Ni}(\mathrm{II})$ catalyst.

Different ligands enable different regioselectivity, a phenomenon which also observed in our previous reductive conditions ${ }^{26,27}$. The mechanism of reductive cross-electrophile coupling of alkyl halides with aryl halides has been thoroughly studied by Weix and coworkers ${ }^{34}$. They have demonstrated that $\mathrm{Ar}-\mathrm{Ni}$ (II) complex was able to react with the alkyl halide, initiating a radical chain process. Thereby, we think the reaction of $\mathrm{Ni}(\mathrm{II})$ complex [Ni]-1 with the alkyl bromide (1b) is also the initiation of the radical chain process in our reaction (Fig. 9g). Moreover, in our prior reductive conditions, the electron-deficient aryl bromides could not efficiently yield the cross-coupling products, but homocoupling products (12) of the aryl bromides were observed instead. We conjecture that $\mathrm{Ar}-\mathrm{Ni}(\mathrm{II})$ complexes [Ni]-2 could also generate in the reactions with $\mathbf{B C}$ ligand, but [Ni]-2 with $\mathbf{1 b}$ was not able to trigger the radical chain, thus leading to the formation of 12 (Fig. 9h). In other words, the selectivity of the products derives from the reactivity of the nickel complexes towards the alkyl electrophiles. The less steric 5,5'-dmbpy (L4) is favorable for subsequent SET. Therefore, $\mathbf{L 4}$ coordinated [Ni]-1 is easier to generate the active $\mathrm{Ni}(\mathrm{III})$ compared with $\mathbf{B C}$ coordinated $[\mathrm{Ni}]-\mathbf{2}$. Instead, the $\mathrm{Ni}(0) / \mathrm{Ni}(\mathrm{II})$ catalytic cycle operates in the reaction with $\mathbf{B C}$ as the ligand. If a stronger oxidant is used, it is supposed to overcome such steric effects and trigger the SET process. This is probably the reason that when more reactive alkyl iodides are used, poor regioselectivity was detected (Fig. 2, 3as). Thus, the selectivity of the final products is determined by the ligand and the reactivity of the substrates. 


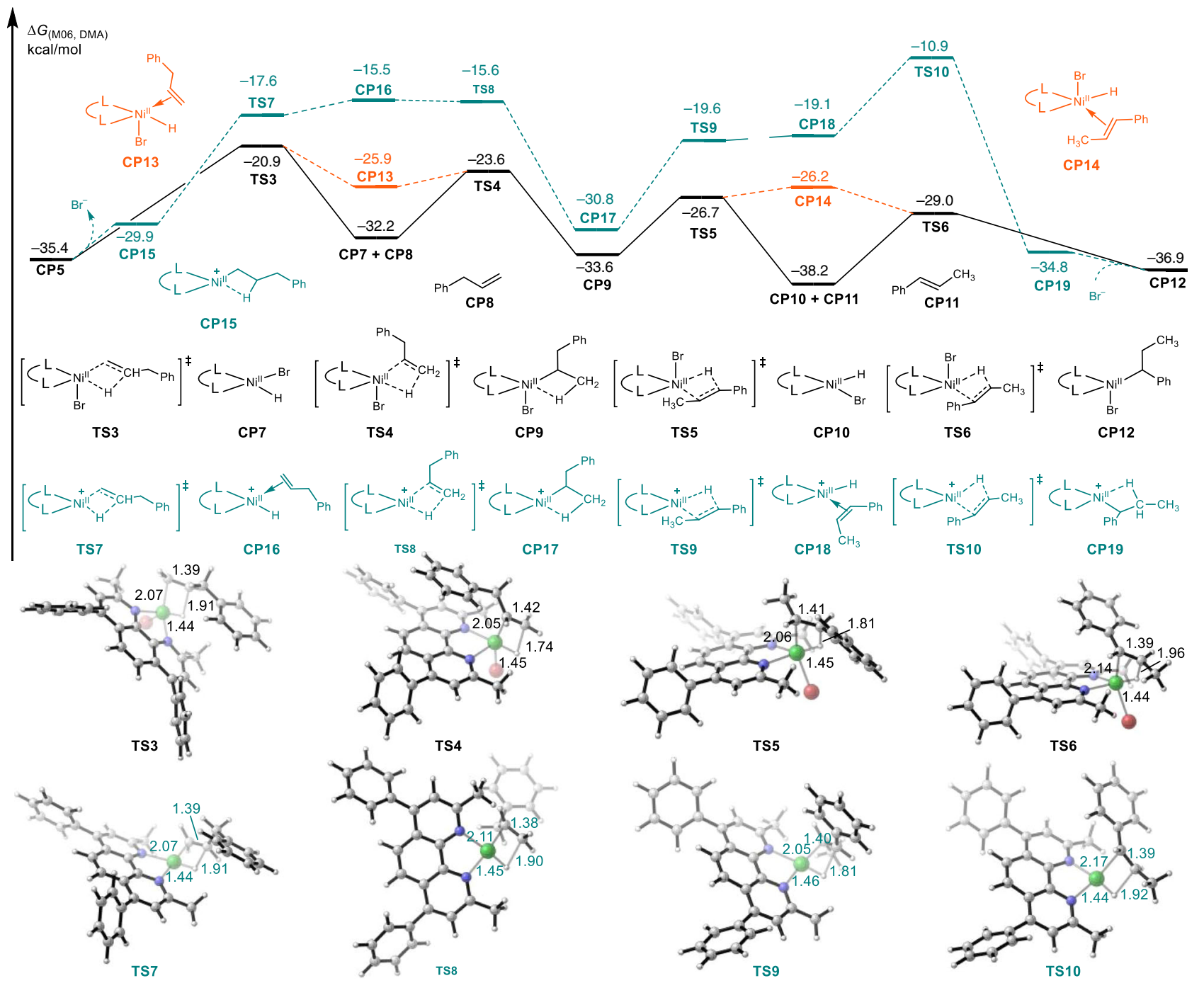

Fig. 7 The calculated reaction energy profile of nickel chain-walking process. Energy values are given in $\mathrm{kcal} / \mathrm{mol}$ and represent the relative free energies calculated by the $\mathrm{M} 06$ method in N, N-dimethylacetamide.

\section{Discussion}

In summary, we have developed a nickel-catalyzed migratory Suzuki-Miyaura cross-coupling reaction, featuring high benzylic or allylic selectivity. This method offers a protocol to rapidly access the diarylalkanes and allylbenzenes from a series of aryl/vinyl-bearing alkyl electrophiles and $\mathrm{sp}^{2}-\mathrm{C}$ boron-based nucleophiles. This approach is characterized by a broad substrate scope and excellent regioselectivity. In addition, the more challenging, but especially useful unactivated alkyl chlorides have been successfully used in the migratory cross-coupling. More potential applications in synthesis are addressed by the combination with selective borylation of $\mathrm{C}-\mathrm{H}$ bonds. Furthermore, this protocol can serve as a platform to access terminal partially deuterium-labeled diarylmethine pharmacophores from the readily accessible precursors.

Mechanistically, the results of Hammett plots suggest that the transmetalation of boronic acid is with a benzyl-Ni(II) species. The DFT calculation suggest that oxidative addition of alkyl halides with $\mathrm{Ni}(0)$ species is prone to involve an SET pathway, the neutral nickel complex is more readily chain-walking than the cationic one, and the olefin is readily dissociated from the catalyst during chain-walking. In addition, the studies towards the original-site cross-coupling reveal that the formation of the product is likely to involve a radical chain process. Both $\mathrm{Ni}(0) / \mathrm{Ni}(\mathrm{II})$ catalytic cycle and radical process are present in these reactions, but the selectivity of the products is controlled by the catalyst and the substrate. We believe this study will greatly advance the future studies toward transformations involving nickel migration.

\section{Methods}

General procedure. Under $\mathrm{N}_{2}$ atmosphere, into an oven-dried $10 \mathrm{~mL}$ reaction tube equipped with a magnetic stir bar and sealed with a rubber stopper sequentially added $\mathrm{NiI}_{2}(15.6 \mathrm{mg}, 0.05 \mathrm{mmol}, 10 \mathrm{~mol} \%)$, BC $(18.0 \mathrm{mg}$, $0.05 \mathrm{mmol}, 10 \mathrm{~mol} \%)$, anhydrous DMA $(4 \mathrm{~mL})$, and $\mathrm{Et}_{3} \mathrm{SiH}(20 \mu \mathrm{L}, 0.13 \mathrm{mmol}$, $25 \mathrm{~mol} \%)$. The mixture was stirred at $35^{\circ} \mathrm{C}$ for $30 \mathrm{~min}$, then TBAB $(161.2 \mathrm{mg}$, $0.5 \mathrm{mmol}, 1.0$ equiv), $\mathrm{LiOH}(29.9 \mathrm{mg}, 1.25 \mathrm{mmol}, 2.5$ equiv), alkyl tosylate $(0.5$ mmol, 1 equiv), and aryl boronic acid $(0.75 \mathrm{mmol}, 1.5$ equiv) were added in this order. The resulting mixture was stirred at $35^{\circ} \mathrm{C}$ (if aryl boronic pinacol ester was used, stirred at $60^{\circ} \mathrm{C}$; if Alkyl bromide was used, TBAB was not need) and monitored by GC until the alkyl tosylate disappeared. After the reaction was complete, the mixture was quenched by saturated brine and extracted with ethyl acetate $(20 \mathrm{~mL} \times 3)$. The combined organic layers were dried over $\mathrm{Na}_{2} \mathrm{SO}_{4}$ and concentrated under reduced pressure. The resulting crude product was separated on a silica gel column affording the cross-coupling product.

\section{Data availability}

The authors declare that the data supporting the findings of this study are available within the article and its Supplementary Information file. The raw data underlying Supplementary Figs. 3 with 55 are provided as a Supplementary Figures file. The raw data underlying Supplementary Tables 1 with 2 are provided as a Supplementary Tables file. The raw data for Absolute Calculation Energies, Enthalpies, and Free Energies are provided as a Source Data file. Additional data are available from the corresponding authors upon request. 


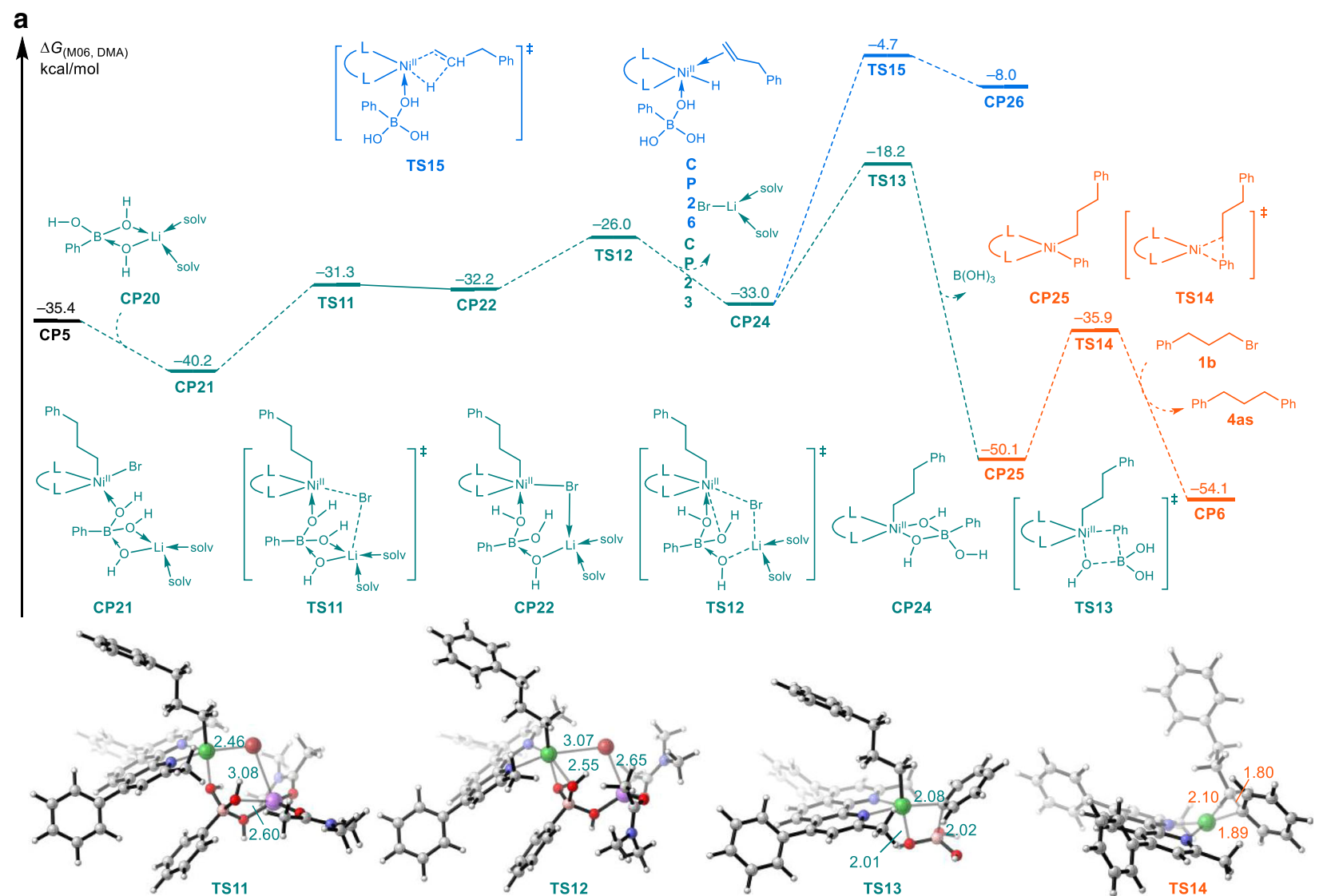

b

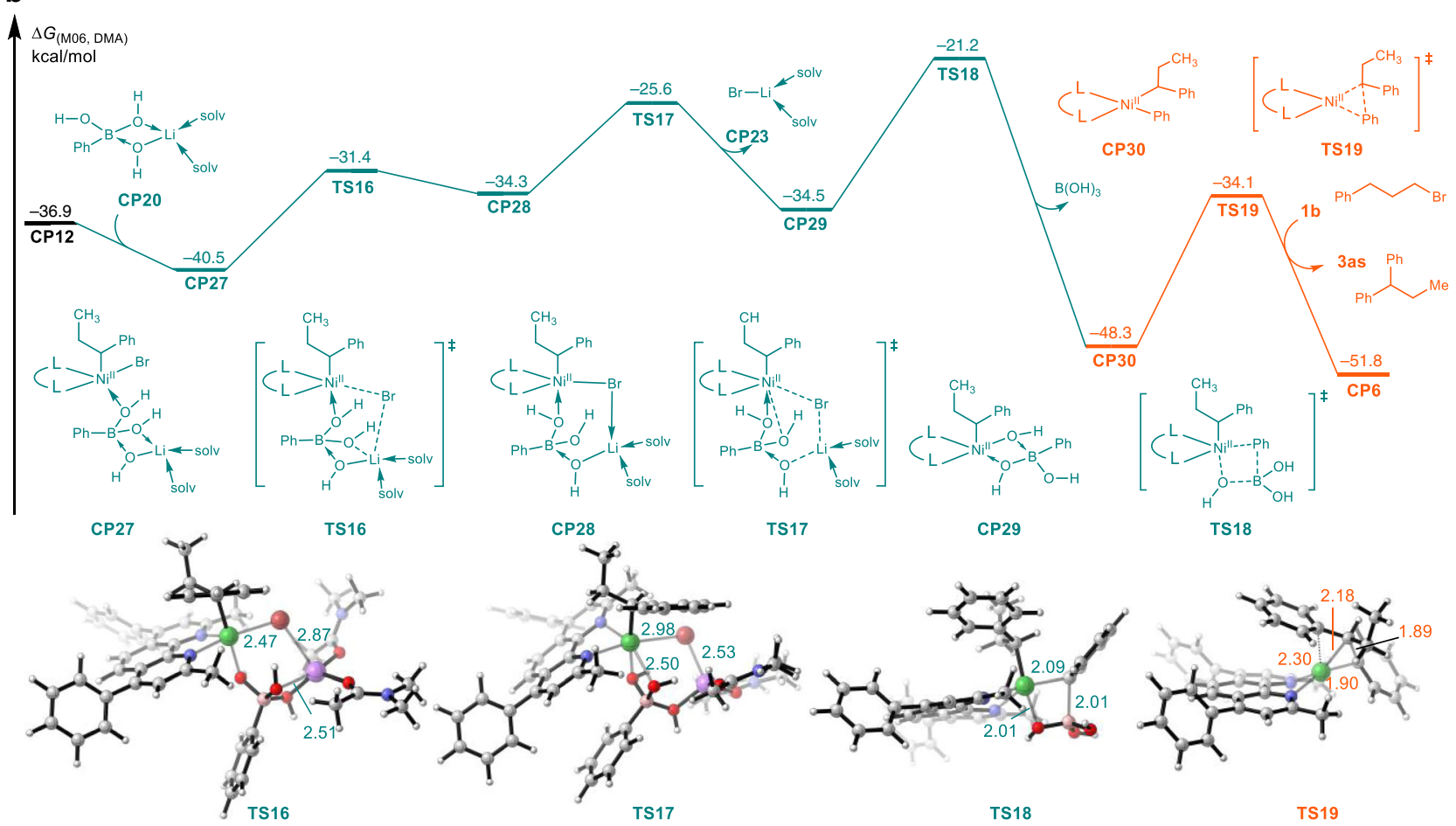

Fig. 8 Calculated reaction energy profile. a Chain-walking and original-site product formation process. Energy values are given in $\mathrm{kcal} / \mathrm{mol}$ and represent the relative free energies calculated by the $\mathrm{M06}$ method in $\mathrm{N}, \mathrm{N}$-dimethylacetamide. The values of bond length are given in ångstrom. $\mathbf{b}$ migratory product formation process. Energy values are given in $\mathrm{kcal} / \mathrm{mol}$ and represent the relative free energies calculated by the M06 method in $\mathrm{N}, \mathrm{N}$-dimethylacetamide. The values of bond length are given in ångstrom. 
a<smiles>N#Cc1ccc([18OH])cc1</smiles>

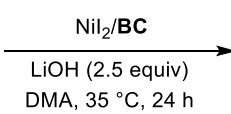

2e

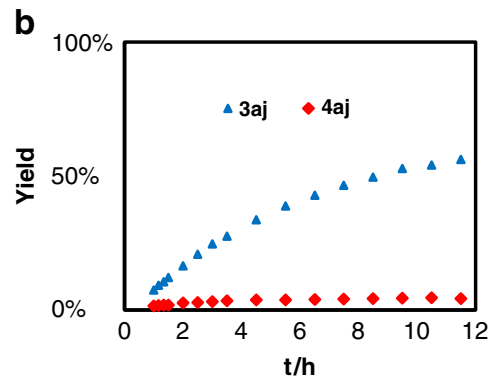

f

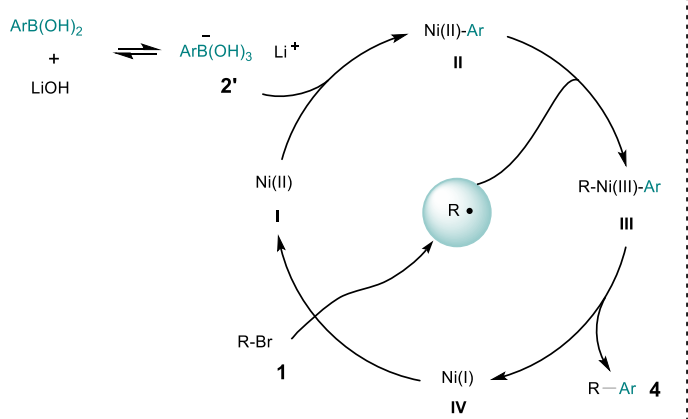

d

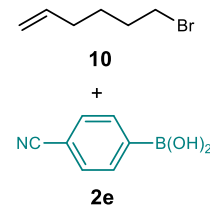

$\frac{\mathrm{Nil}_{2} / \mathrm{L4}(\mathrm{x} \mathrm{mol} \%)}{\mathrm{LiOH}(2.5 \text { equiv })}$

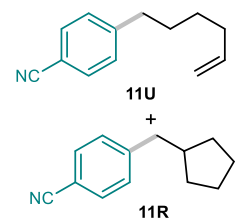

3aj

4aj

C
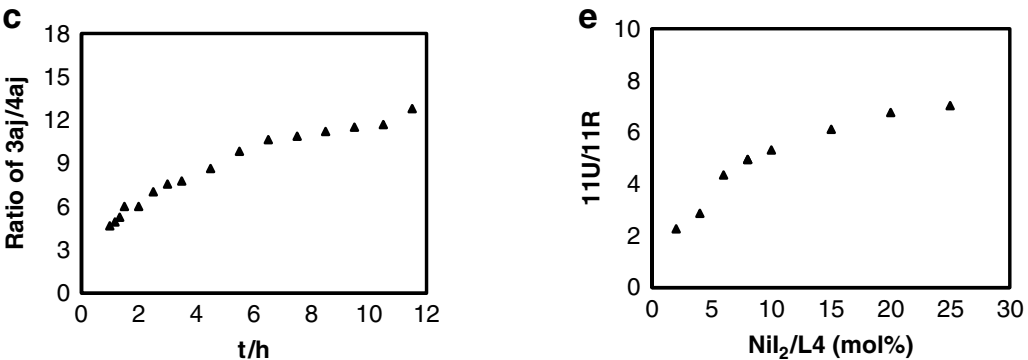

g

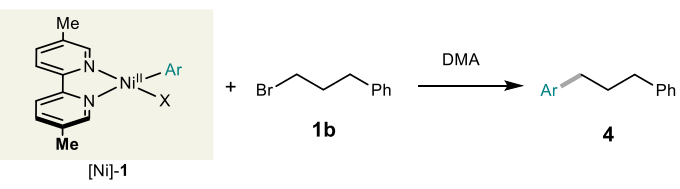

$\mathbf{h}$

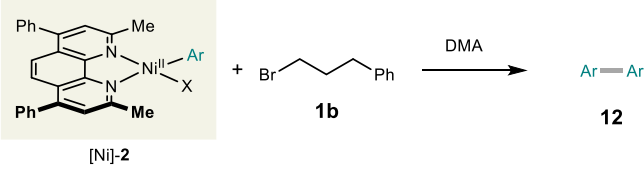

Fig. 9 The study of original-site products. a Model reaction. b Time course of product 3aj and 4aj. c Regioselectivity of migratory product with originalsite product. $\mathbf{d}$ radical clock experiment. $\mathbf{f}$ Effect of catalyst loading on the ratio of $\mathbf{1 1 U} / \mathbf{1 1 R}$. $\mathbf{f}$ Proposed mechanism of original-site product. $\mathbf{g}$ Proposed process on reductive cross-coupling by $\mathrm{Ni} / \mathbf{L} \mathbf{4}$. $\mathbf{h}$ Proposed process on reductive cross-coupling by $\mathrm{Ni} / \mathbf{B C}$.

Received: 10 October 2018; Accepted: 12 December 2019;

Published online: 21 January 2020

\section{References}

1. Liu, C., Zhang, H., Shi, W. \& Lei, A. Bond formations between two nucleophiles: transition metal catalyzed oxidative cross-coupling reactions. Chem. Rev. 111, 1780 (2011).

2. Johansson Seechurn, C. C., Kitching, M. O., Colacot, T. J. \& Snieckus, V. Palladium-catalyzed cross-coupling: a historical contextual perspective to the 2010 nobel prize. Angew. Chem. Int. Ed. 51, 5062 (2012).

3. Colacot, T. New trends in cross-coupling: Theory and applications. RSC Catalysis Series, Royal Society of Chemistry (2015). https://doi.org/10.1039/ 9781782620259

4. Knappke, C. E. et al. Reductive cross-coupling reactions between two electrophiles. Chem. Eur. J. 20, 6828 (2014).

5. Wang, X., Dai, Y. \& Gong, H. Nickel-catalyzed reductive couplings. Top. Curr. Chem. 374, 43 (2016).

6. Terao, J. \& Kambe, N. Cross-coupling reaction of alkyl halides with grignard reagents catalyzed by $\mathrm{Ni}, \mathrm{Pd}$, or $\mathrm{Cu}$ complexes with $\pi$-carbon ligand(s). Acc. Chem. Res. 41, 1545 (2008).

7. Tollefson, E. J., Hanna, L. E. \& Jarvo, E. R. Stereospecific nickel-catalyzed cross-coupling reactions of benzylic ethers and esters. Acc. Chem. Res. 48 , 2344 (2015).

8. Iwasaki, T. \& Kambe, N. Ni-catalyzed C-C couplings using alkyl electrophiles. Top. Curr. Chem. 374, 66 (2016).

9. Kaga, A. \& Chiba, S. Engaging radicals in transition metal-catalyzed crosscoupling with alkyl electrophiles: Recent advances. ACS Catal. 7, 4697 (2017).

10. Netherton, M. R. \& Fu, G. C. Nickel-catalyzed cross-couplings of unactivated alkyl halides and pseudohalides with organometallic compounds. Adv. Synth. Catal. 346, 1525 (2004).
11. Frisch, A. C. \& Beller, M. Catalysts for cross-coupling reactions with nonactivated alkyl halides. Angew. Chem. Int. Ed. 44, 674 (2005).

12. Rudolph, A. \& Lautens, M. Secondary alkyl halides in transition-metalcatalyzed cross-coupling reactions. Angew. Chem. Int. Ed. 48, 2656 (2009).

13. $\mathrm{Hu}, \mathrm{X}$. Nickel-catalyzed cross coupling of non-activated alkyl halides: a mechanistic perspective. Chem. Sci. 2, 1867 (2011).

14. Weix, D. J. Methods and mechanisms for cross-electrophile coupling of $\mathrm{Csp}^{2}$ halides with alkyl electrophiles. Acc. Chem. Res. 48, 1767 (2015)

15. Wang, X., Wang, S., Xue, W. \& Gong, H. Nickel-catalyzed reductive coupling of aryl bromides with tertiary alkyl halides. J. Am. Chem. Soc. 137, 11562 (2015).

16. Wang, X. et al. Ni-catalyzed reductive coupling of electron-rich aryl iodides with tertiary alkyl halides. J. Am. Chem. Soc. 140, 14490 (2018).

17. Vasseur, A., Bruffaerts, J. \& Marek, I. Remote functionalization through alkene isomerization. Nat. Chem. 8, 209 (2016)

18. Sommer, H., Juliá-Hernández, F., Martin, R. \& Marek, I. Walking metals for remote functionalization. ACS Cent. Sci. 4, 153 (2018).

19. Renaudat, A. et al. Palladium-catalyzed $\beta$ arylation of carboxylic esters. Angew. Chem. Int. Ed. 49, 7261 (2010).

20. Baudoin, O. Selectivity control in the palladium-catalyzed cross-coupling of alkyl nucleophiles. Chimia 70, 768 (2016).

21. Dupuy, S., Zhang, K.-F., Goutierre, A.-S. \& Baudoin, O. Terminal-selective functionalization of alkyl chains by regioconvergent cross-coupling. Angew. Chem. Int. Ed. 55, 14793 (2016).

22. Tasker, S. Z., Standley, E. A. \& Jamison, T. F. Recent advances in homogeneous nickel catalysis. Nature 509, 299 (2014)

23. Janssen-Müller, D., Sahoo, B., Sun, S. Z. \& Martin, R. Tackling remote $\mathrm{sp}^{3}$ $\mathrm{C}-\mathrm{H}$ functionalization via Ni-catalyzed "chain-walking" reactions. Isr. J. Chem. 59, 1 (2019)

24. Chen, F. et al. Remote migratory cross-electrophile coupling and olefin hydroarylation reactions enabled by in situ generation of NiH. J. Am. Chem. Soc. 139, 13929 (2017). 
25. He, Y., Cai, Y. \& Zhu, S. Mild and regioselective benzylic C-H functionalization: Ni-catalyzed reductive arylation of remote and proximal olefins. J. Am. Chem. Soc. 139, 1061 (2017).

26. Peng, L. et al. Ligand-controlled nickel-catalyzed reductive relay crosscoupling of alkyl bromides and aryl bromides. ACS Catal. 8, 310 (2017).

27. Peng, L., Li, Z. \& Yin, G. Photochemical nickel-catalyzed reductive migratory cross-coupling of alkyl bromides with aryl bromides. Org. Lett. 20, 1880 (2018).

28. Lu, X. et al. Practical carbon-carbon bond formation from olefns through nickel-catalyzed reductive olefn hydrocarbonation. Nat. Commun. 7, 11129 (2016).

29. Schley, N. D. \& Fu, G. C. Nickel-catalyzed Negishi arylations of propargylic bromides: a mechanistic investigation. J. Am. Chem. Soc. 136, 16588 (2014).

30. Zhao, C., Jia, X., Wang, X. \& Gong, H. Ni-catalyzed reductive coupling of alkyl acids with unactivated tertiary alkyl and glycosyl halides. J. Am. Chem. Soc. 136, 17645 (2014).

31. Knochel, P. Ni(II)-catalyzed cross-coupling between polyfunctional arylzinc derivatives and primary alkyl iodides. J. Am. Chem. Soc. 120, 11186 (1998).

32. Giovannini, R., Studemann, T., Devasagayaraj, A., Dussin, G. \& Knochel, P. New efficient nickel-catalyzed cross-coupling reaction between two Csp ${ }^{3}$ centers. J. Org. Chem. 64, 3544 (1999).

33. Li, Y., Zou, L., Bai, R. \& Lan, Y. Ni(I)-Ni(III) vs. Ni(II)-Ni(IV): Mechanistic study of Ni-catalyzed alkylation of benzamides with alkyl halides. Org. Chem. Front 5, 615 (2018).

34. Biswas, S. \& Weix, D. J. Mechanism and selectivity in nickel-catalyzed crosselectrophile coupling of aryl halides with alkyl halides. J. Am. Chem. Soc. 135, 16192 (2013).

35. Gaydou, M., Moragas, T., Juliá-Hernández, F. \& Martin, R. Site-selective catalytic carboxylation of unsaturated hydrocarbons with $\mathrm{CO}_{2}$ and water. $J$. Am. Chem. Soc. 139, 12161 (2017).

36. Stokes, B. J., Opra, S. M. \& Sigman, M. S. Palladium-catalyzed allylic crosscoupling reactions of primary and secondary homoallylic electrophiles. J. Am. Chem. Soc. 134, 11408 (2012).

37. Stokes, B. J., Bischoff, A. J. \& Sigman, M. S. Pd(quinox)-catalyzed allylic relay suzuki reactions of secondary homostyrenyl tosylates via alkene-assisted oxidative addition. Chem. Sci. 5, 2336 (2014).

38. Lu, Z. \& Fu, G. C. Alkyl-alkyl Suzuki cross-coupling of unactivated secondary alkyl chlorides. Angew. Chem. Int. Ed. 49, 6676 (2010).

39. Atack, T. C. \& Cook, S. P. Manganese-catalyzed borylation of unactivated alkyl chlorides. J. Am. Chem. Soc. 138, 6139 (2016).

40. Borjesson, M., Moragas, T. \& Martin, R. Ni-catalyzed carboxylation of unactivated alkyl chlorides with $\mathrm{CO}_{2}$. J. Am. Chem. Soc. 138, 7504 (2016).

41. Rezazadeh, S., Devannah, V. \& Watson, D. A. Nickel-catalyzed C-alkylation of nitroalkanes with unactivated alkyl iodides. J. Am. Chem. Soc. 139, 8110 (2017).

42. Kirchhoff, J. H., Netherton, M. R., Hills, I. D. \& Fu, G. C. Boronic acids: new coupling partners in room-temperature suzuki reactions of alkyl bromides. Crystallographic characterization of an oxidative-addition adduct generated under remarkably mild conditions. J. Am. Chem. Soc. 124, 13662 (2002).

43. Zhou, J. \& Fu, G. C. Suzuki cross-couplings of unactivated secondary alkyl bromides and iodides. J. Am. Chem. Soc. 126, 1340 (2004).

44. Malhotra, B., Gandelman, K., Sachse, R., Wood, N. \& Michel, M. The design and development of fesoterodine as a prodrug of 5- hydroxymethyl tolterodine (5-Hmt), the active metabolite of tolterodine. Curr. Med. Chem. 16, 4481 (2009).

45. Vanjari, R. \& Singh, K. N. Utilization of methylarenes as versatile building blocks in organic synthesis. Chem. Soc. Rev. 44, 8062 (2015).

46. Rossi, R., Bellina, F. \& Lessi, M. Selective palladium-catalyzed Suzuki-Miyaura reactions of polyhalogenated heteroarenes. Adv. Synth. Catal. 354, 1181 (2012).

47. Murphy, J. M., Liao, X. \& Hartwig, J. F. Meta halogenation of 1,3-disubstituted arenes via iridium-catalyzed arene borylation. J. Am. Chem. Soc. 129, 15434 (2007).

48. Breitenfeld, J., Ruiz, J., Wodrich, M. D. \& Hu, X. Bimetallic oxidative addition involving radical intermediates in nickel-catalyzed alkyl-alkyl kumada coupling reactions. J. Am. Chem. Soc. 135, 12004 (2013).

49. Diccianni, J. B., Katigbak, J., Hu, C. \& Diao, T. Mechanistic characterization of (xantphos)Ni(I)-mediated alkyl bromide activation: oxidative addition, electron transfer, or halogen-atom abstraction. J. Am. Chem. Soc. 141, 1788 (2019).

50. Dudnik, A. S. \& Fu, G. C. Nickel-catalyzed coupling reactions of alkyl electrophiles, including unactivated tertiary halides, to generate carbon-boron bonds. J. Am. Chem. Soc. 134, 10693 (2012).

51. Lu, Z., Wilsily, A. \& Fu, G. C. Stereoconvergent amine-directed alkyl-alkyl suzuki reactions of unactivated secondary alkyl chlorides. J. Am. Chem. Soc 133, 8154 (2011).
52. Atzrodt, J., Derdau, V., Kerr, W. J. \& Reid, M. Deuterium- and tritiumlabelled compounds: applications in the life sciences. Angew. Chem. Int. Ed. 57, 1758 (2018)

53. Wang, X. et al. General and practical potassium methoxide/disilane-mediated dehalogenative deuteration of (hetero)arylhalides. J. Am. Chem. Soc. 140, 10970 (2018)

54. Xia, H.-M., Zhang, F.-L., Ye, T. \& Wang, Y.-F. Selective $\alpha$-monomethylation by an amine-borane/ $\mathrm{N}, \mathrm{N}$-dimethylformamide system as the methyl source. Angew. Chem. Int. Ed. 57, 11770 (2018).

55. Huang, C.-Y. \& Doyle, A. G. Nickel-catalyzed Negishi alkylations of styrenyl aziridines. J. Am. Chem. Soc. 134, 9541 (2012).

56. Verheyen, T. et al. Ketone synthesis by a nickel-catalyzed dehydrogenative cross-coupling of primary alcohols. J. Am. Chem. Soc. 141, 6869 (2019).

57. Yi, Y.-Q.-Q. et al. Nickel-catalyzed C-N bond reduction of aromatic and benzylic quaternary ammonium triflates. Chem. Commun. 52, 10894 (2016).

58. KC, $\mathrm{S}$. et al. Ni-catalyzed regioselective alkylarylation of vinylarenes via $\mathrm{C}$ $\left(\mathrm{sp}^{3}\right)-\mathrm{C}\left(\mathrm{sp}^{3}\right) / \mathrm{C}\left(\mathrm{sp}^{3}\right)-\mathrm{C}\left(\mathrm{sp}^{2}\right)$ bond formation and mechanistic studies. J. Am. Chem. Soc. 140, 9801 (2018).

59. Wang, Z., Yin, H. \& Fu, G. C. Catalytic enantioconvergent coupling of secondary and tertiary electrophiles with olefins. Nature 563, 379 (2018).

60. Ni, S. et al. A radical approach to anionic chemistry: synthesis of ketones, alcohols, and amines. J. Am. Chem. Soc. 141, 6726 (2019).

61. Jin, L., Xin, J., Huang, Z., He, J. \& Lei, A. Transmetalation is the rate-limiting step: quantitative kinetic investigation of nickel-catalyzed oxidative coupling of arylzinc reagents. J. Am. Chem. Soc. 132, 9607 (2010).

62. Xu, L. et al. Mechanism, reactivity, and selectivity in palladium-catalyzed redox-relay Heck arylations of alkenyl alcohols. J. Am. Chem. Soc. 136, 1960 (2014).

63. Kapat, A., Sperger, T., Guven, S. \& Schoenebeck, F. E-olefins through intramolecular radical relocation. Science 363, 391 (2019).

64. de Aguirre, A., Funes-Ardoiz, I. \& Maseras, F. Four oxidation states in a single photoredox Ni-based catalytic cycle: a computational study. Angew. Chem. Int. Ed. 58, 3898 (2019).

65. Omer, H. M. \& Liu, P. Computational study of Ni-catalyzed C-H functionalization: factors that control the competition of oxidative addition and radical pathways. J. Am. Chem. Soc. 139, 9909 (2017).

66. Singh, S. K. S. \& Sunoj, R. B. Aliphatic $\mathrm{C}\left(\mathrm{sp}^{3}\right)-\mathrm{H}$ bond activation using nickel catalysis: mechanistic insights on regioselective arylation. J. Org. Chem. 82, 9619 (2017).

\section{Acknowledgements}

We thank Profs Qianghui Zhou, Wen-Bo Liu, and Aiwen Lei at Wuhan University for lending lab space and sharing the basic instruments. We are grateful for the financial support from National Natural Science Foundation of China (21702151, 21871211, 21822303, and 21703159) and the Fundamental Research Funds for Central Universities (2042019kf0208, 2018CDXZ0002 and 2018CDPTCG0001/4). We thank Dr Joshua Buss at University of Wisconsin-Madison for his very helpful suggestions on the manuscript preparation.

\section{Author contributions}

Yuqiang Li designed and carried out most of the chemical reactions and analyzed the data. Long Peng, Yangyang Li, Binzhi Zhao, Wang Wang, Hailiang Pang, and Yi Deng supported the design and performance of synthetic experiments. Guoyin Yin designed and supervised the project. Yixin Luo, Ruopeng Bai, and Yu Lan conducted the calculations. Yuqiang Li, Yixin Luo, Ruopeng Luo, Yu Lan, and Guoyin Yin wrote the manuscript. All authors discussed the results and commented on the manuscript, and approved the final version of the manuscript.

\section{Competing interests}

The authors declare no competing interests.

\section{Additional information}

Supplementary information is available for this paper at https://doi.org/10.1038/s41467 019-14016-1.

Correspondence and requests for materials should be addressed to Y.L. or G.Y.

Peer review information Nature Communications thanks Donghui Wei and the other, anonymous, reviewer(s) for their contribution to the peer review of this work. Peer reviewer reports are available.

Reprints and permission information is available at http://www.nature.com/reprints

Publisher's note Springer Nature remains neutral with regard to jurisdictional claims in published maps and institutional affiliations. 
(c) (i) Open Access This article is licensed under a Creative Commons Attribution 4.0 International License, which permits use, sharing, adaptation, distribution and reproduction in any medium or format, as long as you give appropriate credit to the original author(s) and the source, provide a link to the Creative Commons license, and indicate if changes were made. The images or other third party material in this article are included in the article's Creative Commons license, unless indicated otherwise in a credit line to the material. If material is not included in the article's Creative Commons license and your intended use is not permitted by statutory regulation or exceeds the permitted use, you will need to obtain permission directly from the copyright holder. To view a copy of this license, visit http://creativecommons.org/ licenses/by/4.0/.

(C) The Author(s) 2020 